\title{
A Programmable TEM Image Collection System
}

\author{
N. Nakamura*, T. Moriya*, T. Shinkawa*, C.R. Booth** and W. Chiu** \\ *JEOL Ltd., 1-2, Musashino 3-chome, Akishima, Tokyo 196-8558 Japan \\ **National Center for Macromolecular Imaging, Baylor College of Medicine, Houston, TX 77030
}

There have been many efforts to develop computerized methods to acquire data using TEM. These methods increase the rate at which researchers can acquire data. For example, automated acquisition of tilted images have freed TEM users who are working on tomography from repetitive operations $[1,2,3,4,5]$; automatic specimen search and acquisition on ice-embedded specimens have helped researchers study single particles in a high throughput manner [6,7]; and automatic sample screening has reduced a need to sit in front of a TEM for hours to screen for potential crystalline specimens [8].

Existing systems are limited by their specificity for a single type of data collection, and are not well suited to other modes of operation. These systems may be flexible in the way they evaluate each acquired image $[6,7,8]$. However, all of these systems have strong limitations on the way data is collected. Researchers may use the same microscope with different imaging protocols. For example, one may collect tomographic data one day and a low dose focal pair on another. Until now there is no software package reported that can allow the user to readily design data collection schemes that meet their various research objectives.

We have been developing software that provides a platform to perform flexible, high throughput, high quality data collection for the present generation of JEOL transmission electron microscopes. To address this, we have built a software system that provides elements of TEM control that the users can arrange in a sequence to suit their own purposes. The TEM control elements are available that perform high level microscope functions such as auto-focusing, and searching for specimens as well as low level functions such as adjusting lens and deflector values. The user can arrange these elements in a sequence to suit the experiment being conducted. This "recipe" can be combined with one of three modes of operations (Figure 1): automatic, semi-automatic, and manual to create a novel experimental protocol, which is useful for high throughput, high quality data collection.

As an initial proof of the principle, we have designed this system to be utilized especially for collecting images of ice embedded single particles by cryo-TEM. This software, however, should be useful for different protocols other than those for ice embedded single particles. The automatic search operation provides automatic hole detection and positioning, ice thickness evaluation, focusing and image acquisition. This algorithm iterates over all of the grid squares initially identified by the user. Semi-automatic search algorithm requires the user to identify the holes or areas within a grid square suitable for imaging. This procedure is useful not only with holey carbon film but also with objects 
located on continuous carbon film. Finally, the manual operation can be executed similar to a standard low dose method. The user controls the stage to locate an area of the specimen to be imaged and commands the software to carry out the sequence of focus and photo operations.

\section{References}

[1] Motoki, S. et al., 8APEM, Kanazawa, Japan, 178-179 (2004).

[2] Mastronarde, D.N., Microsc and Microanal 9: 1182-1183 (2003).

[3] Rath, B.K. et al., J Struct Biol, 120:210-218 (1997).

[4] Ziese, U. et al., J Microsc, 205:187-200, (2002).

[5] Koster, A.J. et al., J Struct Biol, 120: 276-308 (1997).

[6] Carragher, B. et al., Microsc and Microanal, 7: 970-971 (2001).

[7] Zhang, P. et al., J Struct Biol, 135: 251-261 (2001).

[8] Nakamura, N. et al., Microsc and Microanal, 9: 1038-1039 (2003).

[9] This research was partially supported by grants from NIH P41RR02250 and Robert Welch Foundation to W. Chiu and C. Booth.
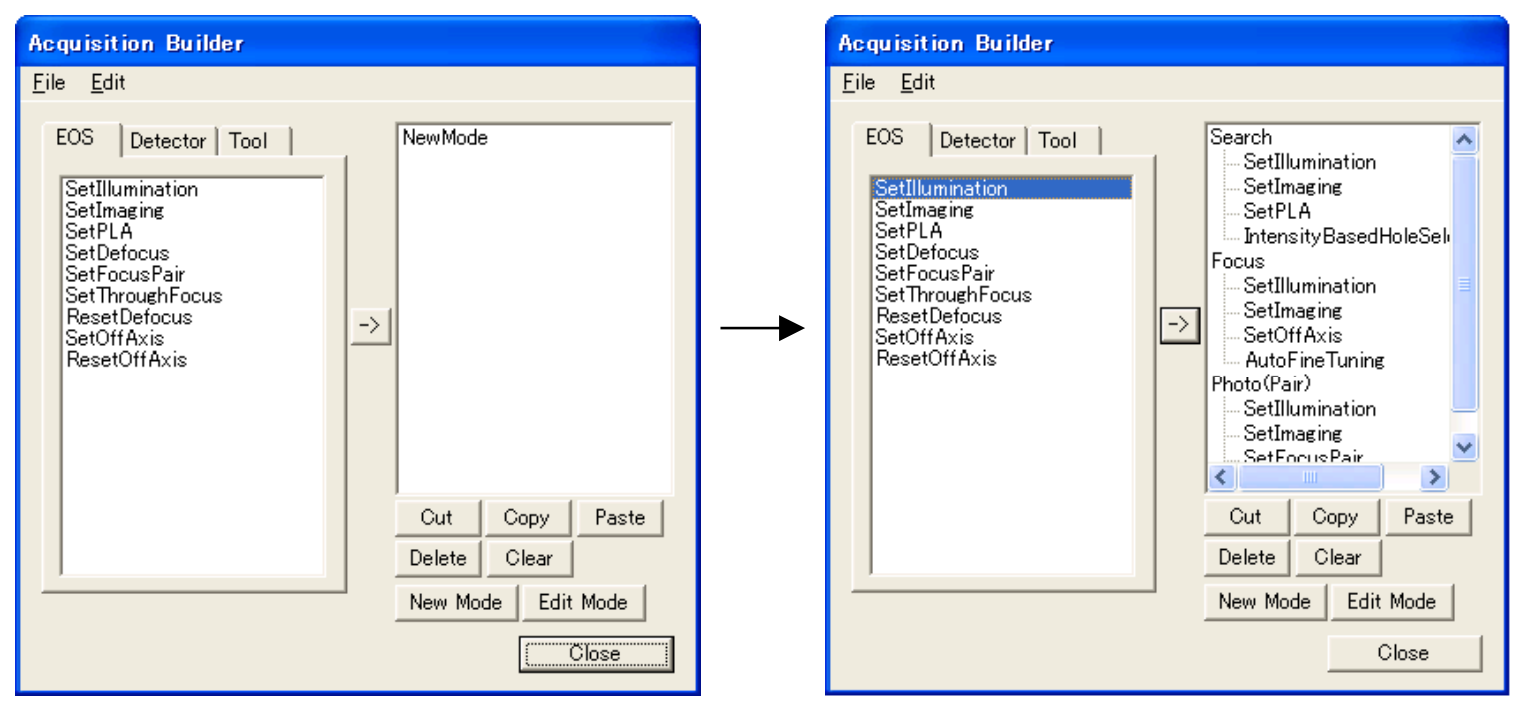

Fig. 1. The user-interface for "recipe" programming. This example shows how to build a sequence of focal pair acquisition with MDS (Minimum Dose System). 